Document downloaded from:

http://hdl.handle.net/10251/178932

This paper must be cited as:

Jimenez, N.; Benlloch Baviera, JM.; Camarena Femenia, F. (2020). A new elastographic technique using acoustic vortices. IEEE. 1-4.

https://doi.org/10.1109/IUS46767.2020.9251417

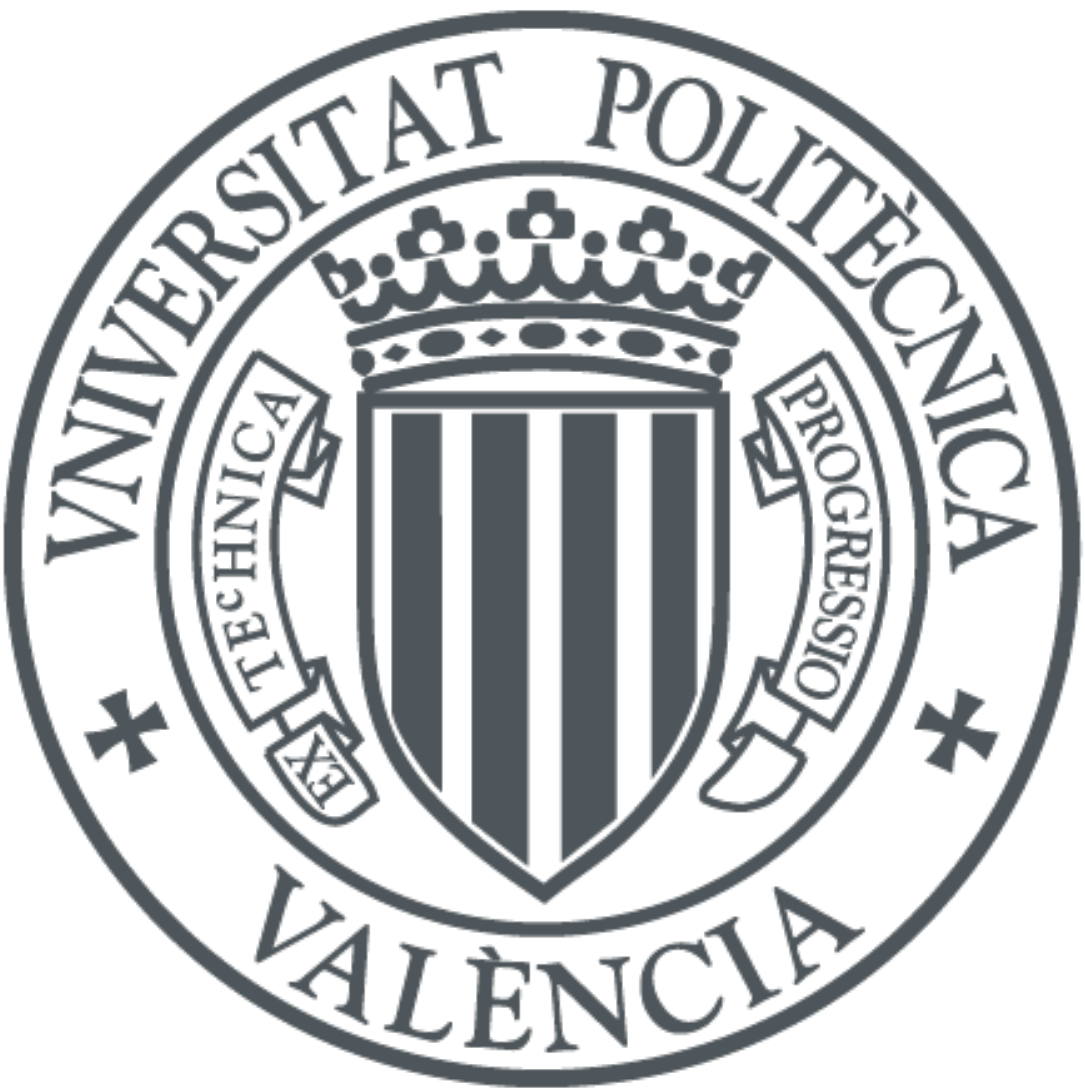

The final publication is available at

https://doi.org/10.1109/IUS46767.2020.9251417

Copyright IEEE

Additional Information 


\title{
A new elastographic technique using acoustic vortices
}

\author{
Noé Jiménez*, José María Benlloch and Francisco Camarena \\ Instituto de Instrumentación para Imagen Molecular \\ Universitat Politècnica de València - CSIC, València, Spain \\ *nojigon@upv.es
}

\begin{abstract}
We present a novel method of elastography based on acoustic vortices to transfer angular momentum to tissue in addition to linear momentum. Focused vortex beams can push and twist tissue, and the rotation direction of the applied torque can be dynamically controlled by the modulation of the topological charge of the vortex. The technique results in a robust excitation of shear waves with quasi-omnidirectional radiation pattern and arbitrary waveform, which may have a great impact in imaging performance for elastography.

Index Terms-Elastography, Acoustic Vortex, Acoustic Radiation Force, Acoustic Torque
\end{abstract}

\section{INTRODUCTION}

During last decades ultrasound elastography has been developed as a reliable technique for medical diagnosis [1]. Commonly, tissues are deformed under the action of the acoustic radiation forces by using a primary-pushing acoustic beam as a result of the transfer of linear momentum. Then, tissue displacements are quantified by ultrasound imaging, enabling in this way multiple elastography modalities [2]-[6]. However, using focused beams the acoustic radiation force can only push the tissue in the direction of the propagation of the beam and the degree of freedom to displace the tissue in other directions is limited.

Beyond the linear momentum carried by focused ultrasound beams, acoustic vortex beams carry angular orbital momentum [7]. Acoustic vortex beams are wavefronts containing phase dislocations as $\exp (i l \phi)$, with $\phi$ the azimuthal angle and $l$ the topological charge. The field presents a phase singularity at the axis of the beam, exhibiting at this point a null caused by destructive interferences. It has been demonstrated that the angular momentum carried by an acoustic vortex beams can be transferred to micro and macroscopic solid objects [8] or viscous fluids [9], exerting mechanical torques to these media. In addition, vortex beams induce trapping acoustic radiation forces on small particles, leading to particle tweezing and manipulation systems [10]-[12]. These acoustic vortices can exert mechanical forces several orders of magnitude higher than those of their optical counterparts, with lower induced

This research has been supported by the Spanish Ministry of Science, Innovation and Universities through grants "Juan de la Cierva - Incorporación" IJC2018-037897-I and PID2019-111436RBC22, and by the Agencia Valenciana de la Innovació through grants INNVA1/2020/92 and INNCON/2020/009. Action cofinanced by the European Union through the Programa Operativo del Fondo Europeo de Desarrollo Regional (FEDER) of the Comunitat Valenciana 2014-2020 (IDIFEDER/2018/022). thermal effects [13], and, furthermore, acoustic vortices can propagate deeply through opaque media such as biological tissues. Acoustic vortices have recently shown their potential for biomedical applications to manipulate individual cells without inducing photothermal and/or photochemical damages [14], to trap and guide kidney stones fragments in-vivo [15], or to selectively guide individual drug-delivery carriers based on microbubbles [16].

As phase dislocations are at the basis of these techniques, the wavefront must be controlled with accuracy. Several methods to synthesize vortex beams have been reported, including active sources or phased arrays systems [17], transducers with helical geometries [17], [18], Archimedean [19] or Fresnel spiral diffraction gratings [20], systems exploiting the photoacoustic effect [21], active vibrating surfaces with spiral shape [22], or metamaterials exhibiting local resonances [23], [24]. Of special interest for wavefront engineering are acoustic holograms [25]. By synthesising complex wavefronts, acoustic holograms can efficiently compensate skull aberrations and, simultaneously, conform arbitrary acoustic images adapting to the desired therapeutic target [26]. Acoustic holograms can be used to generate vortex beams with great accuracy by encoding the full-wavefront information into an array or a 3D printed lens [27], [28].

We present a novel method of elastography based on acoustic vortices to transfer to tissues, in addition to linear, angular momentum. By controlling the topological charge, this patented method [29] efficiently deforms the tissue with a great degree of freedom, i.e., modulating the displacements waveforms or controlling the polarization of the shear waves.

\section{Methods}

A focused vortex beam is generated by a ring-shaped piezoelectric focused source (H105, Sonic Concepts), with a focal of $F=64 \mathrm{~mm}$, and outer and inner apertures of $64 \mathrm{~mm}$ and $37 \mathrm{~mm}$, respectively, and a central frequency of $f=1.1$ MHz. An helicoidal phase-plate lens that allows the selection of the topological charge of the vortex was 3D printed in photo-reactive polymer by stereolithography (Form2, Formlabs), and coupled with vacuum grease to the matching layer of the transducer. The lens was manufactured with a single topological charge, $l=1$, as shown in Figs. $1(\mathrm{a}, \mathrm{b})$. A 64-elements phased-array piezoelectric probe (H105, Sonic Concepts) is mounted concentrically. 

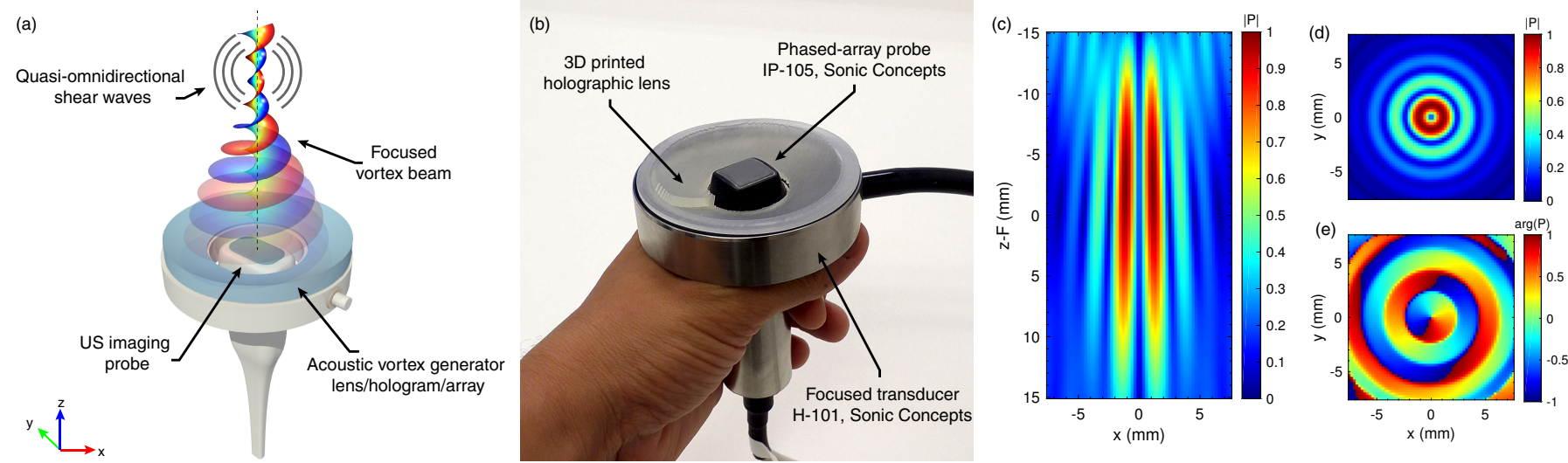

Fig. 1. (a) Conceptual design of vortex-elastography systems, where the vortex generator can be implemented by a lens, an hologram or a phased-array probe. (b) Implementation using a 3D printed lens for the vortex generator and a 64-elements phased array probe for the imaging system. (c) Magnitude of the calculated pressure field in the $p=p(x, 0, z)$ plane. (d) Magnitude and (e) phase of the calculated pressure field in the transverse $p=p(x, y, F)$ plane.

The acoustic field produced by the lens at the exit plane of the transducer, $P=P\left(\mathbf{r}_{\mathbf{0}}\right)$ is calculated in the frequency domain by projecting the field of the focused source multiplied by a phase dislocation of topological charge of $l$ times as

$$
P\left(\mathbf{r}_{0}, \omega\right)=p_{0} \exp \left(-i k \sqrt{x_{0}^{2}+y_{0}^{2}+F^{2}}\right) \exp (i l \theta),
$$

where $k=\omega / c_{0}$ is the acoustic wavenumber, $c_{0}$ is the sound speed, $\omega=2 \pi f$ is the angular frequency, and $\theta$ is the azimuthal coordinate. The acoustic field at any point of the space $P=P(\mathbf{r}, \omega)$ far from the source, $\mathbf{r} \neq \mathbf{r}_{0}$, is given by the Rayleigh diffraction integral

$$
P(\mathbf{r}, \omega)=\frac{-i \omega \rho_{0}}{2 \pi} \int_{S} \frac{V_{0}\left(\mathbf{r}_{0}\right) \exp \left(i k\left|\mathbf{r}-\mathbf{r}_{0}\right|\right)}{\left|\mathbf{r}-\mathbf{r}_{0}\right|} d S,
$$

where $V_{0}\left(\mathbf{r}_{0}\right) \approx P\left(\mathbf{r}_{0}\right) / \rho_{0} c_{0}$, and $\rho_{0}$ is the medium density. Once the acoustic field is known, and up to second order approximation, the acoustic radiation (body) force is given by [30]

$$
\mathbf{F}(\mathbf{r}, \omega)=i \frac{\alpha(\omega)}{2 \omega \rho_{0} c_{0}}\left(P \nabla P^{*}-P^{*} \nabla P\right),
$$

where $\alpha(\omega)$ is the absorption coefficient of the ultrasound waves at the angular frequency $\omega$.

Finally, to model the displacements, following Ref. [30], a pseudo-spectral time-domain simulation method [31] was used to solve the equations

$$
\begin{aligned}
\frac{\partial \sigma_{i j}}{\partial t} & =\lambda \delta_{i j} \frac{\partial v_{k}}{\partial x_{k}}+\mu\left(\frac{\partial v_{i}}{\partial x_{j}}+\frac{\partial v_{j}}{\partial x_{i}}\right), \\
\frac{\partial v_{i}}{\partial t} & =\frac{1}{\rho_{0}} \frac{\partial \sigma_{i j}}{\partial x_{j}}+\frac{1}{\rho_{0}} F_{i}(\mathbf{r}, t),
\end{aligned}
$$

where $\lambda$ and $\mu$ are the Lame's parameters of the soft solid, and $\sigma_{i j}=\sigma_{i}(\mathbf{r}, t)$ and $v_{i}=v_{i}(\mathbf{r}, t)$ are the $i$-th component of the stress and particle velocity in the soft solid, respectively, and $\delta_{i j}$ is the Kronecker delta function, and $F_{i}(\mathbf{r}, t)$ is the temporal waveform of the force modulated by the envelope of the ultrasound beam pulse.

\section{RESULTS}

First, the field of the primary beam generated by the lens in water, calculated using (2), shows a phase dislocation on the axis and a topological charge $l=1$, as shown in Figs. 1 (ce). The system presents a linear gain of 16.0 and the distance between maxima, i.e., the with of the toroidal focus, is $1.7 \mathrm{~mm}$.

Second, the absorbing tissue phantom of $0.5 \mathrm{~dB} / \mathrm{cm} / \mathrm{MHz}$ is introduced and a force appears as a result of an energy density gradient. The acoustic radiation force is calculated using (3) in the soft tissue model. The components of the force are shown in Figs. 2 (a-e). In Fig. 2 (a) the axial component in the plane $(x, 0, z)$, is plotted normalized $\left(F_{z} / F_{\max }\right)$, and the normalized lateral components of the force in the plane $(x, y, F), F_{x} / F_{\max }$ and $F_{y} / F_{\max }$, are shown in Figs. $2(\mathrm{~b}, \mathrm{c})$, respectively. Finally, the acoustic radiation torque exerted in the tissue in the plane $(x, y, F), F_{\theta} / F_{\max }$ is shown in Fig. 2 (d) and Fig. 2 (e) shows the direction of the resulting force in the transverse plane $(x, y, F)$. It is shown that for this focusing, the rotational component is about 3 times smaller than the axial component. Sharper focusing or higher topological charges can improve this ratio.

Under a transient ultrasound pulse of duration $\tau=3 \mathrm{~ms}$, equivalent to 3300 cycles at $1.1 \mathrm{MHz}$, and assuming a shear wave speed of $2 \mathrm{~m} / \mathrm{s}$, pulsed shear waves are generated. The spatial distribution of the tissue displacements is shown in Fig. 3 at different instants of time. A transient shear wave is generated and propagates transversally to the source in the $x$ direction. As angular momentum is transferred to the medium at the focal spot, this results in a point source of torsional shear waves that quasi-omnidirectionally propagate from the focus.

Finally, vortex elastography is used for shearwave dispersion ultrasound vibrometry [32]. A sine-sweep modulated signal is applied to the primary ultrasound emitter as

$$
p_{0}(t)=\sin \left(2 \pi\left[\frac{f_{1}-f_{0}}{2 T} t^{2}+f_{0} t\right] t\right) \sin \left(\omega_{0} t\right),
$$



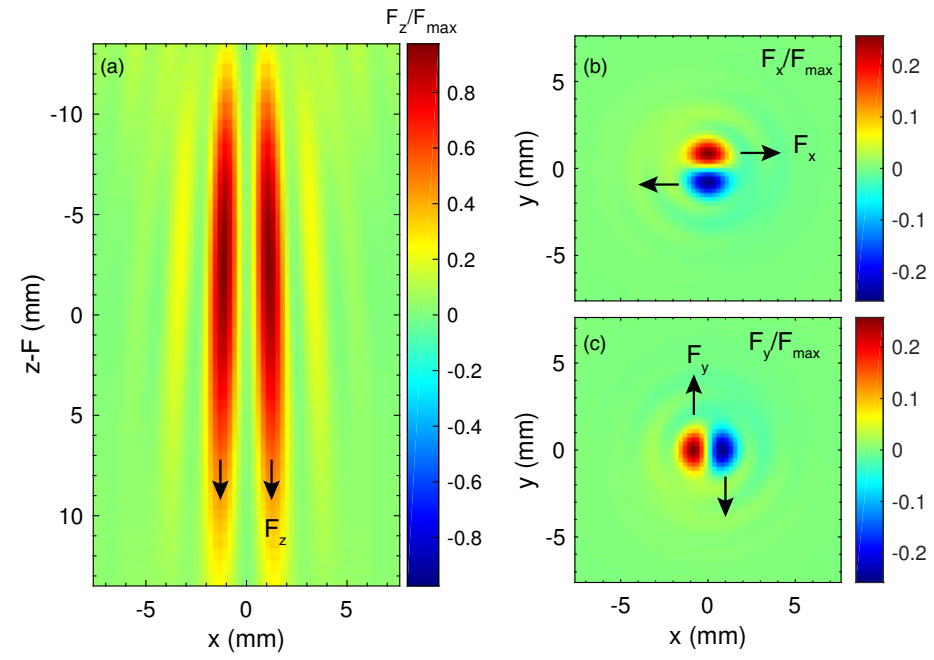

where $f_{0}=150 \mathrm{~Hz}$ and $f_{1}=800 \mathrm{~Hz}$ are the minimum and maximum frequencies, and $T=37 \mathrm{~ms}$ is the duration of the sine-sweep pulse. In addition, the polarity of the topological charge is modulated using a phased-array vortex emitter in the simulation as

$$
l(t)=l_{0} \operatorname{sign}\left[\sin \left(2 \pi\left[\frac{f_{1}-f_{0}}{2 T} t^{2}+f_{0} t\right] t\right)\right]
$$

where $l_{0}=4$ is the magnitude of the topological charge, as shown in Figs $4(\mathrm{a}, \mathrm{b})$. The displacements are measured along the $x$ direction, a sample is shown in Fig. 4 (c), where it can be observed the sine-sweep waveform retrieved in the simulations for the displacements in the $y$ direction. Then, the spatio-temporal data is transformed to the $k-\omega$ frequency domain using a 2D Fast Fourier Transform. In this way the dispersion relation of the phantom is obtained. The modes corresponding to the theoretical dispersion relation, given for this elastic phantom by the linear function $\omega(k)=c_{0} k$, are shown by the colour map in Fig. 4 (d). It can be observed that the reconstructed modes belongs to the theoretical dispersion relation, marked as a white dashed line.

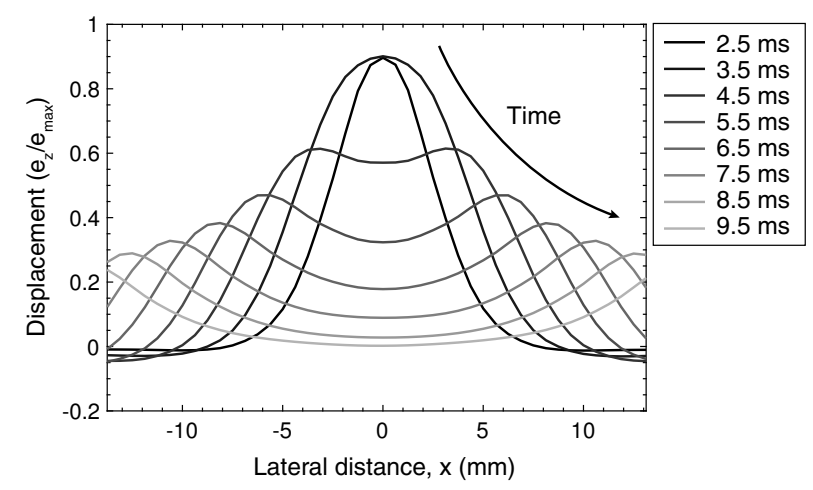

Fig. 3. Simulated displacements in the axial direction $\left(e_{z} / e_{\max }\right)$ at different instants of time, as a function of the lateral direction $(x)$.

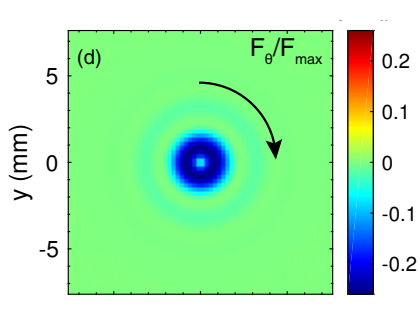

Fig. 2. Acoustic radiation force calculated from the acoustic field in the soft tissue model. (a) Axial component in the plane $(x, 0, z)$, normalized $\left(F_{z} / F_{\max }\right),(\mathrm{b}, \mathrm{c})$ normalized lateral components in the plane $(x, y, F), \quad F_{x} / F_{\max }$ and $F_{y} / F_{\max }$, respectively. (d) Acoustic radiation torque exerted in the tissue in the plane $(x, y, F)$, $F_{\theta} / F_{\max }$ and (e) vector field in

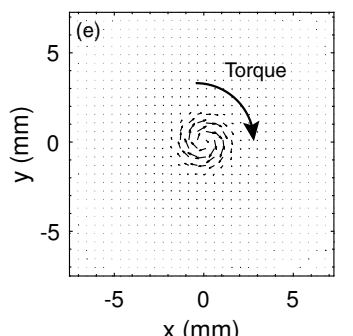
the plane $(x, y, F)$ showing the direction of the resulting force in the transverse plane.

\section{Conclusions}

We have presented a new method of elastography based on the transfer of orbital angular momentum from a vortex beam to soft tissues, either using a lens or a phased-array to generate the vortex beam. With this method the tissue can be twisted, in addition to pushed as it happens with conventional systems. Then, by time modulating the ultrasound beam, shear waves can be efficiently generated. Finally, by tracking the shear waves the elasticity of the medium can be obtained in a robust way. In addition, by the temporal modulation of the sign of the topological charge of the vortex, e.g., by using a phased-array to synthesize the vortex beam, the direction of rotation of the torque can be controlled. Thus, the forced excitation can be harmonic or follow an arbitrary waveform, as a sine-sweep excitation, enabling the efficient generation of shear waves of high amplitude with controlled polarization. This technique results in a robust excitation of shear waves with quasi-omnidirectional radiation pattern and arbitrary waveform, which have a great potential to improve the imaging quality of ultrasound elastography.

\section{REFERENCES}

[1] I. Z. Nenadic, M. W. Urban, J. F. Greenleaf, J.-L. Gennisson, M. Bernal, and M. Tanter, Ultrasound elastography for biomedical applications and medicine. John Wiley \& Sons, 2019.

[2] A. P. Sarvazyan, O. V. Rudenko, S. D. Swanson, J. B. Fowlkes, and S. Y. Emelianov, "Shear wave elasticity imaging: a new ultrasonic technology of medical diagnostics," Ultrasound in medicine \& biology, vol. 24, no. 9, pp. 1419-1435, 1998.

[3] L. Sandrin, B. Fourquet, J.-M. Hasquenoph, S. Yon, C. Fournier, F. Mal, C. Christidis, M. Ziol, B. Poulet, F. Kazemi et al., "Transient elastography: a new noninvasive method for assessment of hepatic fibrosis," Ultrasound in medicine \& biology, vol. 29, no. 12, pp. 1705-1713, 2003.

[4] K. R. Nightingale, M. L. Palmeri, R. W. Nightingale, and G. E. Trahey, "On the feasibility of remote palpation using acoustic radiation force," The Journal of the Acoustical Society of America, vol. 110, no. 1, pp. 625-634, 2001.

[5] E. E. Konofagou and K. Hynynen, "Localized harmonic motion imaging: theory, simulations and experiments," Ultrasound in medicine \& biology, vol. 29, no. 10, pp. 1405-1413, 2003. 


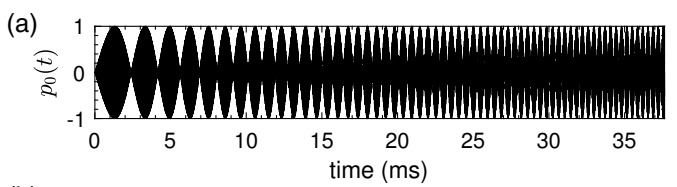

(b)
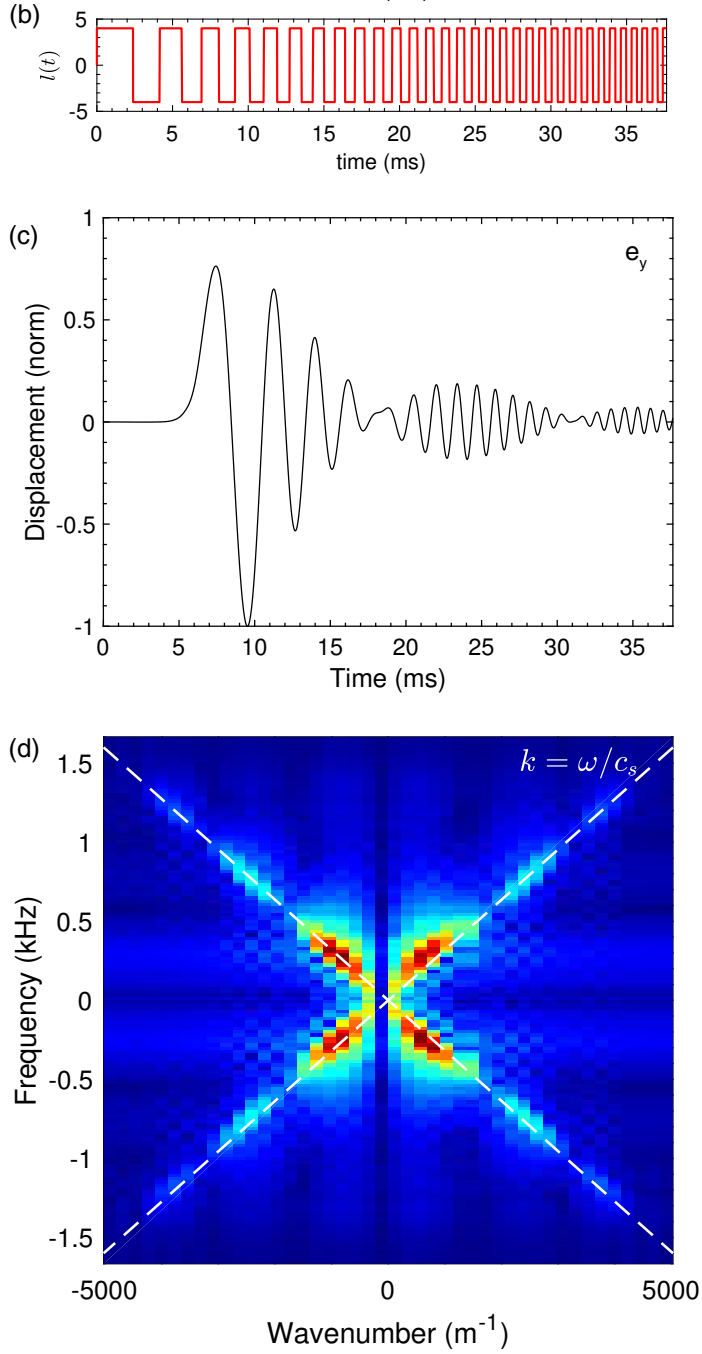

Fig. 4. Example of application of the modulation of the topological charge to obtain the elastic properties of the medium as a function of the frequency. (a) Modulation signal for the primary field. (b) Modulation signal for the topological charge. (c) Simulated displacement in the $y$ direction retrieved at $y=0, x=2 \mathrm{~mm}$ and $z=F$. (d) (color map) Dispersion relation obtained by double FFT of the temporal signals along the $x$ direction, and (white dashed) theoretical dispersion relation of the phantom.

[6] J. Bercoff, M. Tanter, and M. Fink, "Supersonic shear imaging: a new technique for soft tissue elasticity mapping," IEEE transactions on ultrasonics, ferroelectrics, and frequency control, vol. 51, no. 4, pp. 396-409, 2004.

[7] J.-L. Thomas and R. Marchiano, "Pseudo angular momentum and topological charge conservation for nonlinear acoustical vortices," Physical review letters, vol. 91, no. 24, p. 244302, 2003.

[8] K. Volke-Sepúlveda, A. O. Santillán, and R. R. Boullosa, "Transfer of angular momentum to matter from acoustical vortices in free space," Phys. Rev. Lett., vol. 100, no. 2, p. 024302, 2008.

[9] A. Riaud, M. Baudoin, J.-L. Thomas, and O. B. Matar, "Cyclones and attractive streaming generated by acoustical vortices," Physical Review E, vol. 90, no. 1, p. 013008, 2014.

[10] J. Wu, "Acoustical tweezers," Jour. Acous. Soc. Am., vol. 89, no. 5, pp.
2140-2143, 1991.

[11] D. Baresch, J.-L. Thomas, and R. Marchiano, "Observation of a singlebeam gradient force acoustical trap for elastic particles: acoustical tweezers," Physical review letters, vol. 116, no. 2, p. 024301, 2016.

[12] A. Marzo, M. Caleap, and B. W. Drinkwater, "Acoustic virtual vortices with tunable orbital angular momentum for trapping of mie particles," Physical review letters, vol. 120, no. 4, p. 044301, 2018.

[13] D. G. Grier, "A revolution in optical manipulation," Nature, vol. 424, no. 6950, p. 810,2003

[14] M. Baudoin, J.-L. Thomas, R. A. Sahely, J.-C. Gerbedoen, Z. Gong, A. Sivery, O. Matar, N. Smagin, and A. Vlandas, "Cell selective manipulation with single beam acoustical tweezers," arXiv preprint arXiv:2001.04162, 2020.

[15] M. A. Ghanem, A. D. Maxwell, Y.-N. Wang, B. W. Cunitz, V. A. Khokhlova, O. A. Sapozhnikov, and M. R. Bailey, "Noninvasive acoustic manipulation of objects in a living body," Proceedings of the National Academy of Sciences, 2020.

[16] D. Baresch and V. Garbin, "Acoustic trapping of microbubbles in complex environments and controlled payload release," Proceedings of the National Academy of Sciences, vol. 117, no. 27, pp. 15 490-15 496, 2020.

[17] B. T. Hefner and P. L. Marston, "An acoustical helicoidal wave transducer with applications for the alignment of ultrasonic and underwater systems," Jour. Acous. Soc. Am., vol. 106, no. 6, pp. 3313-3316, 1999.

[18] J. L. Ealo, J. C. Prieto, and F. Seco, "Airborne ultrasonic vortex generation using flexible ferroelectrets," IEEE transactions on ultrasonics, ferroelectrics, and frequency control, vol. 58, no. 8, pp. 1651-1657, 2011.

[19] N. Jiménez, R. Picó, V. Sánchez-Morcillo, V. Romero-García, L. M. García-Raffi, and K. Staliunas, "Formation of high-order acoustic bessel beams by spiral diffraction gratings," Physical Review E, vol. 94, no. 5, p. 053004, 2016.

[20] N. Jiménez, V. Romero-García, L. M. García-Raffi, F. Camarena, and K. Staliunas, "Sharp acoustic vortex focusing by fresnel-spiral zone plates," Applied Physics Letters, vol. 112, no. 20, p. 204101, 2018.

[21] S. Gspan, A. Meyer, S. Bernet, and M. Ritsch-Marte, "Optoacoustic generation of a helicoidal ultrasonic beam," Jour. Acous. Soc. Am., vol. 115, no. 3, pp. 1142-1146, 2004.

[22] R. D. Muelas-Hurtado, J. L. Ealo, J. F. Pazos-Ospina, and K. VolkeSepúlveda, "Generation of multiple vortex beam by means of active diffraction gratings," Applied Physics Letters, vol. 112, no. 8, p. 084101, 2018.

[23] X. Jiang, Y. Li, B. Liang, J.-c. Cheng, and L. Zhang, "Convert acoustic resonances to orbital angular momentum," Phys. Rev. Lett., vol. 117, no. 3, p. 034301, 2016.

[24] L. Ye, C. Qiu, J. Lu, K. Tang, H. Jia, M. Ke, S. Peng, and Z. Liu, "Making sound vortices by metasurfaces," AIP Advances, vol. 6, no. 8, p. 085007, 2016.

[25] K. Melde, A. G. Mark, T. Qiu, and P. Fischer, "Holograms for acoustics," Nature, vol. 537, no. 7621, pp. 518-522, 2016.

[26] S. Jiménez-Gambín, N. Jiménez, J. M. Benlloch, and F. Camarena, "Holograms to focus arbitrary ultrasonic fields through the skull," Physical Review Applied, vol. 12, no. 1, p. 014016, 2019.

[27] A. Marzo, S. A. Seah, B. W. Drinkwater, D. R. Sahoo, B. Long, and S. Subramanian, "Holographic acoustic elements for manipulation of levitated objects," Nature communications, vol. 6, p. 8661, 2015.

[28] S. Jiménez-Gambín, N. Jiménez, J. M. Benlloch, and F. Camarena, "Generating bessel beams with broad depth-of-field by using phase-only acoustic holograms," Scientific Reports, vol. 9, no. 1, pp. 1-13, 2019.

[29] N. Jiménez, J. M. Benlloch, and F. Camarena, "Procedimiento de obtención de propiedades elásticas de un sólido blando que hace uso de vórtices acústicos," Spanish Patent 201930675, Jun. 2019.

[30] F. Prieur and O. A. Sapozhnikov, "Modeling of the acoustic radiation force in elastography," The Journal of the Acoustical Society of America, vol. 142, no. 2, pp. 947-961, 2017.

[31] K. Firouzi, B. Cox, B. Treeby, and N. Saffari, "A first-order k-space model for elastic wave propagation in heterogeneous media," The Journal of the Acoustical Society of America, vol. 132, no. 3, pp. 1271$1283,2012$.

[32] M. W Urban, S. Chen, and M. Fatemi, "A review of shearwave dispersion ultrasound vibrometry (SDUV) and its applications," Current Medical Imaging, vol. 8, no. 1, pp. 27-36, 2012. 